\title{
Feasibility of recycling concrete construction waste in environmental and economic sides
}

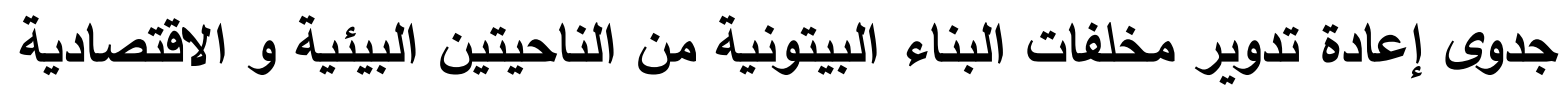

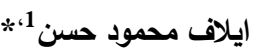

1 كلية الهندسة المدنية ـقسم الهندسة البيئية ، جامعة البعث، سوريا.

* Corresponding author: Elbagir Eltayeb (e-mail: elaf.hasan2511@gmail.com).

Article history: Received 23 May 2020, Received in revised form 10 April 2021, Accepted 12 November 2021

Digital Object Identifier (doi): https://doi.org/10.52981/fjes.v11i1.503

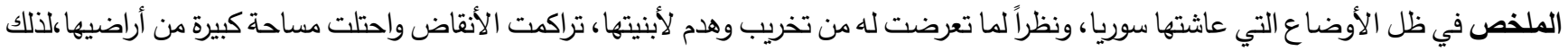

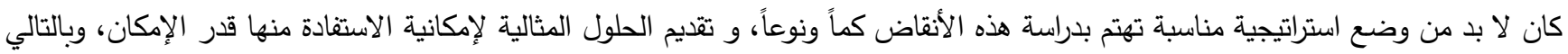

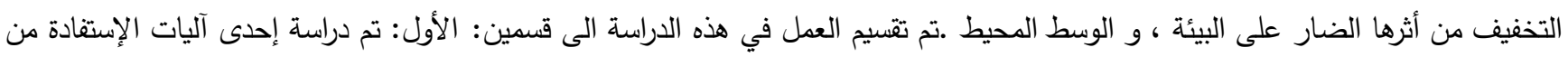

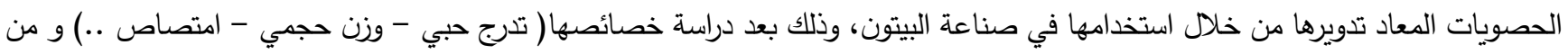

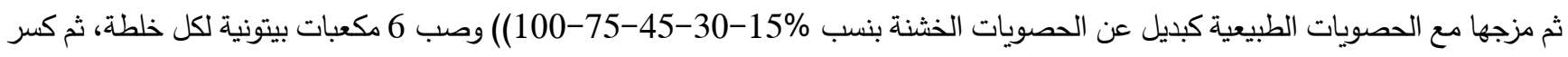
نصف عدد العينات على عمر 7 ايام و النصف الاخر على عمر 28 يوماً .حيث تم استتاج العلاقة بين نسبة الحصويات المعاد تدويرها و المقاومة

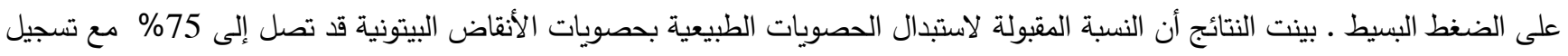

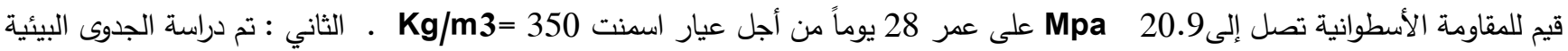

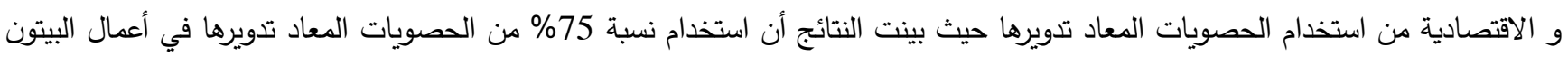

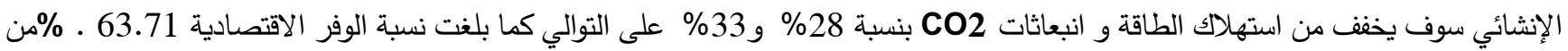
خلال هذه الدراسة يمكن تحقيق غايتين : الأولى إزالة كميات كبيرة من مصادر التلوث البيئي الناتج عن هذه المخلفات، و بالتالي تحقيق غاية بيئية .

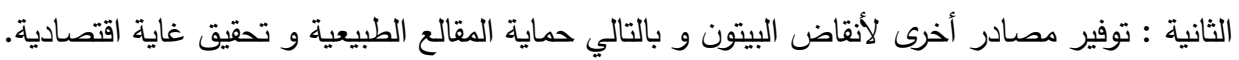

الكلمات المفتاحية الحصويات المعاد تدويرها - ركام البيتون - المقاومة على الضغط البسيط - التشرب - الجدوى البيئية و الاقتصادية .

ABSTRACT In light of the conditions experienced by Syria and due to the destruction and demolition of its buildings built up rubble and occupied a large area of its territory. Therefore, it was necessary to develop an appropriate strategy to study these debris and provide the ideal solutions to be able to benefit from them as much as possible and thus mitigate the harmful impact on the environment and the surrounding environment. The work is divided into two parts: First: The study focuses on one of the mechanisms of benefiting from the recycled aggregate by using them in producing concrete, after studying their characteristics(Sieve Analysis-density- Absorption) and then mixing them with natural aggregate as replacement of natural coarse aggregate at different rates(15-30-45-75-100)\%. Six concrete cubes were made for each mixture and 3 cubes were broken after 7 days and the other after 28 days. it was concluded The relationship between the percentage of recycled aggregates and compressive strength, as well as the relationship between the percentage of stones and the rate of Absorption The results showed that the acceptable percentage of replacement of natural aggregates with recycled aggregates may reach $100 \%$ with the recording of values of resistance of up to $21.9 \mathrm{Mpa}$. Second :The environmental and economic feasibility of using recycled aggregates has been studied the results showed that use $75 \%$ of the recycled aggregates in structural concrete works will reduce 
energy consumption and carbon dioxide emissions by $28 \%$ and $33 \%$, respectively, and the economic saving rate is $63.71 \%$. Through this study two objectives can be achieved: First: removing large quantities of environmental pollution sources resulting from these wastes and thus achieving an environmental goal Second: Provide other sources of concrete aggregates and thus protect the natural quarries and achieve an economic goal.

Keywords: Recycled aggregate -Concrete aggregate - compressive strength - Absorptionenvironmental and economic feasibility.

\section{1}

الثكل (2) دورة حياة مواد البناء

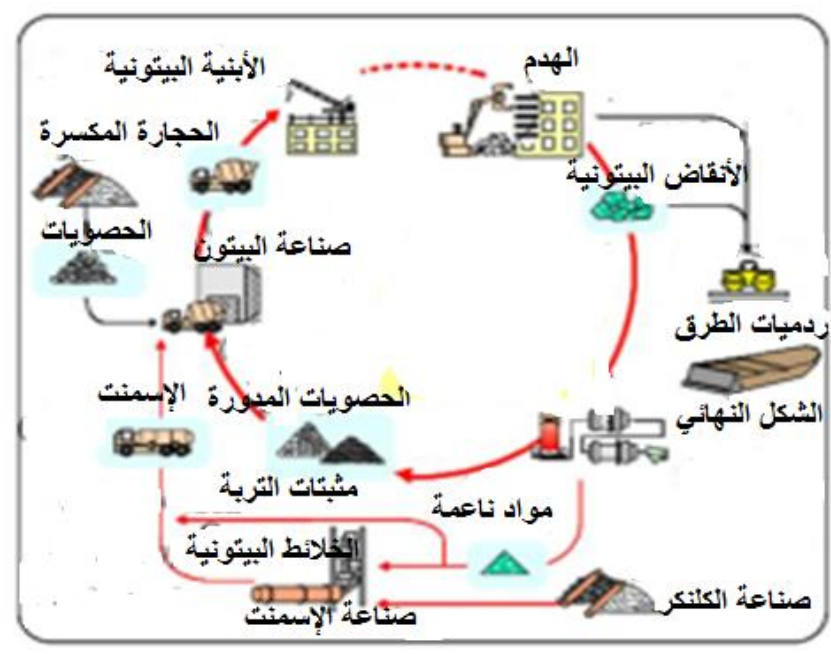

الثكل (3) منهجية إعادة تدوير البيتون[4]

2. الأضرار التي تخلفها أنقاض مواد البناء:

1 -ملء المطامر الصحية بثكل سريع.

2 -تلوث الهواء و زيادة نسبة الرمال والغبار فيه، و بالتالي تلوث البيئة

و إلحاق الضرر بالروح البشرية.

3 -احتمال تلوث المياه الجوفية (حيث أنه بعد تعرض مخلفات المباني

لمياه الأمطار في أماكن تكسها، تذوب كميات كبيرة من سيلكات الكالسيوم

المززوجة بالمياه وهيدروكسيد الكالسيوم الموجودة في قطع البيتون، وكميات

كبيرة من أيونات الكبريت الموجودة في مخلفات الجبس، وكميات كبيرة من

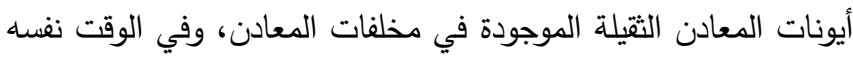

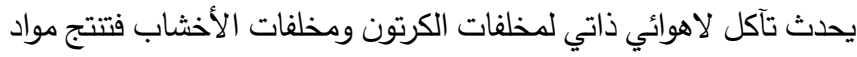

تتحلل إلى أحماض عضوية، وهذه المواد المنتجة تتسرب إلى المياه فتصبح

شديدة القلوية وتحتوي على كمية كبيرة من أيونات المعادن الثقيلة وكبريتيد الهيدروجين وكمية معينة من المواد العضوية، مما قد يؤدي إلى تلوث المياه

$$
\text { فوق سطح الأرض والمياه الجوفية. }
$$

يعتبر البيتون من أهم مواد البناء لما له من ميزات إيجابية، ولا سيما كونه

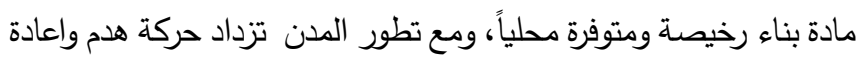

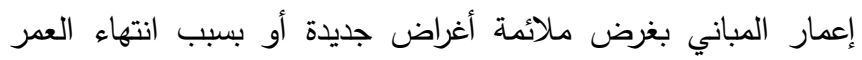

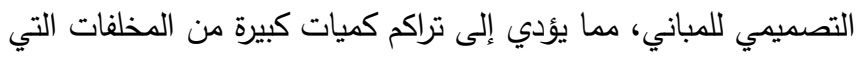

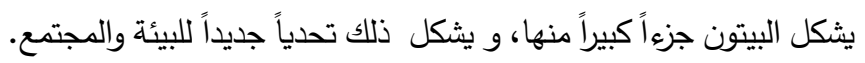

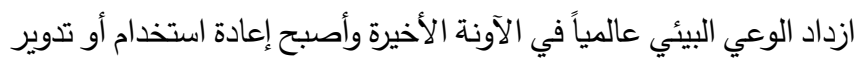

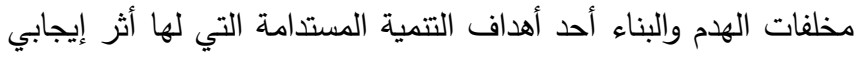

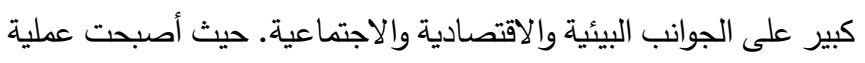
إعادة التدوير سياسة معتمدة في الكثير من الدول المتقدمة، وتعتبر هولندا

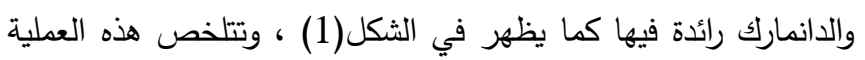

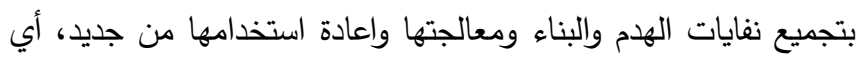

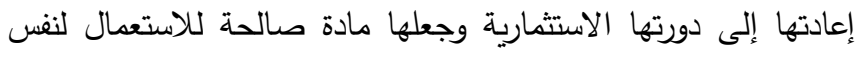
الغرض أو لأغراض أخرى كما في الثكل(2). وهذه الطريقة مفيدة بيئياً

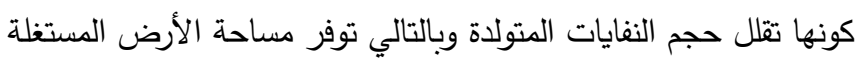
كمكب للنفايات، كما أنها تساهم في الحفاظ على الدصادر الطبيعية

الأصلية.

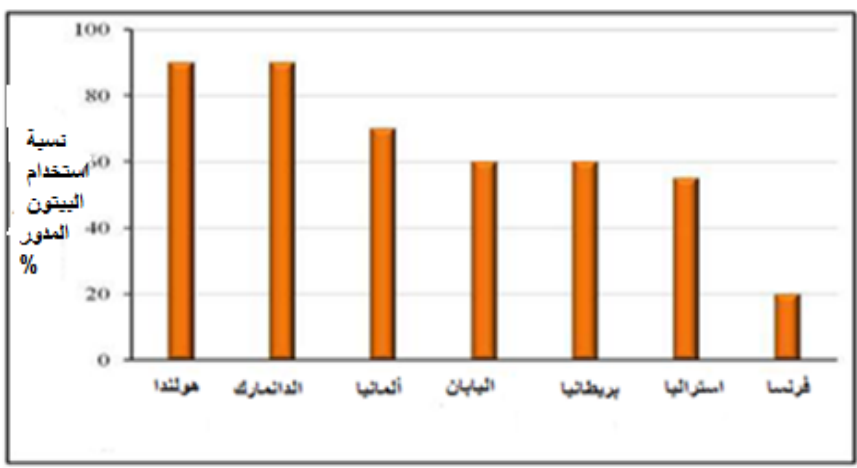

الثكل (1) النسب المئوية لإعادة تدوير البيتون في دول العالم[1]

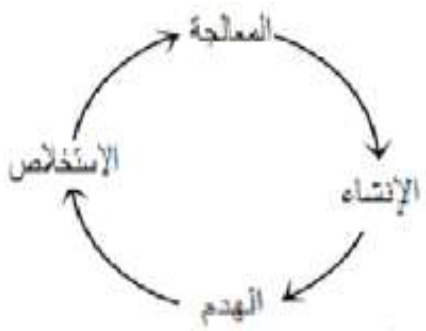




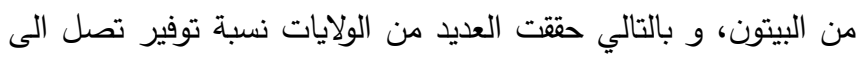

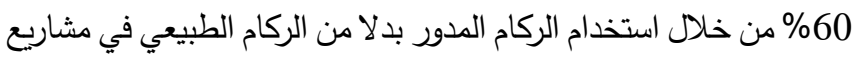

في استراليا انخفضت التكاليف السنوية بحدود 85\% عند استخدام الركام المدور ، وذلك بسبب إلغاء رسوم المكب و انخفاض التكاليف

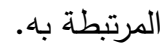

بالنسبة للبلدان الاقل نمواً، أقيمت دراسة في تايلند بينت أن استخدام 100\% من الركام المدور يمكن أن يؤدي إلى إنتاج كتل بيتونية

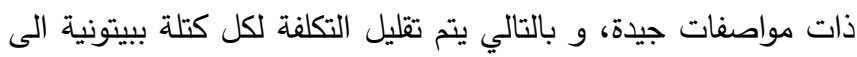

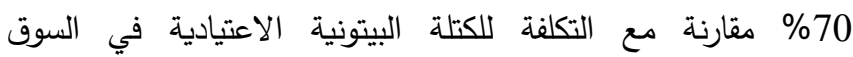
التايلندية [5]. 6 -توفير فرص عمل واستثمار من خلال إنثاء مصانع إعادة تدوير

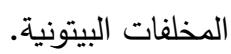
7-تحسين صورة البلد امام الرأي العالم العالمي.

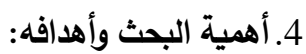

يعتبر البيتون في قطاع البناء و التشييد من مواد البناء الأكثر استخداماً،

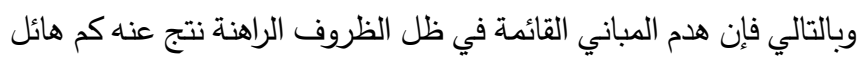

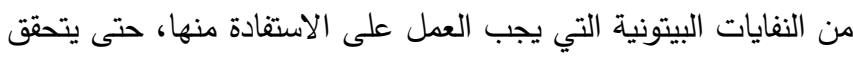

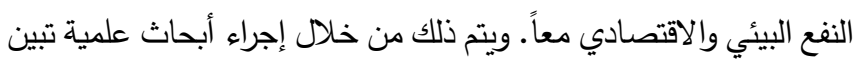
مدى إمكانية الاستفادة من هذه النفايات، وبالتالي يتوفر بيانات ومعطيات

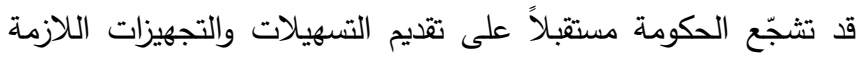

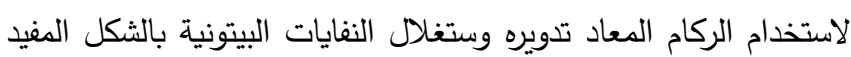

والصحيح.

ويبقى السبب الأكبر لتراكم الأنقاض في بلادنا هو الإرهاب الذي

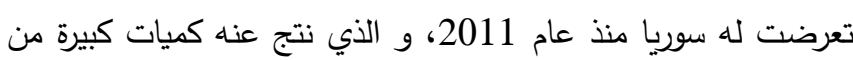

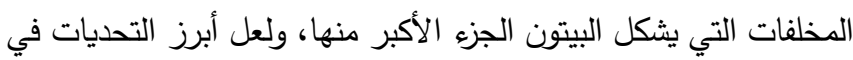

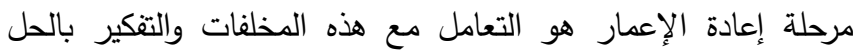
للاستفادة منها بدلاً من إرسالها الى الدكبات وتثكيل كارثة بيئية خطيرة

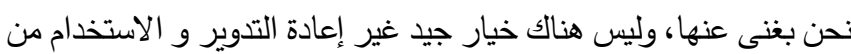

\section{يهدف هذا البحث إلى} 1

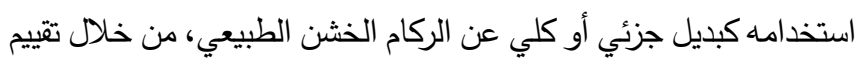

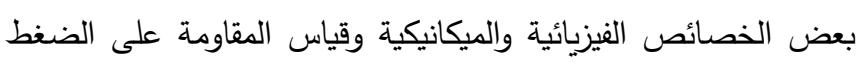
البسيط ، وإجراء مقارنة لخصائص هذا البيتون مع البيتون التقليدي. 2 -تحديد الأثر البيئي الناتج من إعادة تدوير مخلفات البناء البيتونية.

$$
3 \text {-دراسة الجدوى الاقتصادية للبحث. }
$$

تندرج أهمية البحث تحت ثلاث نقاط رئيسية:
4-تأخذ حيزاً كبيراً من الأمكنة الضرورية لإنشاء ساحات أوشوارع

$$
\text { أوحتى ممرات يستفاد منها. }
$$

5 -كلف عالية للإزالة و التنظيف.

6-قد تكون عرضة لعبث الأطفال مما يشكل خطراً عليهم.

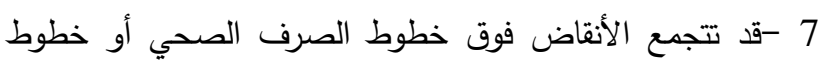

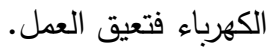

8 -تأثيرات اقتصادية حيث أكدت الدراسات أن المخلفات الإنشائية هي

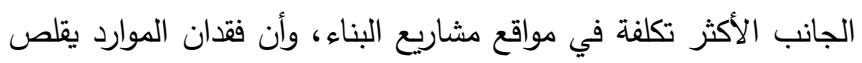
الأرباح ويزيد التكاليف على المالك ، وإذا كانت مواد البناء تمثل نسبة فئل 50\% فإن فقدان 20\% من هذه المواد يتسبب في خسارة 10\% من الكلفة الإجمالية للمشروع ، وتصبح هذه النسبة في منتهى الخطورة عندما يتعلق

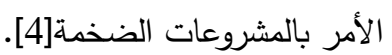
** "في البلدان العربية يأخذ الموضوع أهمية استثنائية خاصة في ظل الطروف التي نعيش فيها من الحروب و الأزمات التي خلفت مئات الاطنان

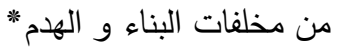

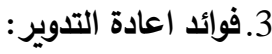

إن عملية التشييد و البناء بطبيعتها ليست صديقة للبيئة لذلك إعادة التدوير فعالة جداً في معالجة هذه المشكلة ولها فوائد بيئية و اقتصادية كبيرة منها: 1 -الحفاظ على الطبيعة حيث أن استخدام الحصويات المدورة يقلل من اللجوء إلى الحصويات الطبيعية من المقالع. فعلى سبيل المثال، في

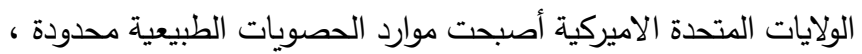
مما دفع إلى استخدام الحصويات المدورة على نطاق واسع كحل اقتصادي وصديق للبيئة [2] . 2 -تحسين البيئة العامة عن طريق تقليل صناعة التعدين، و بالتالي

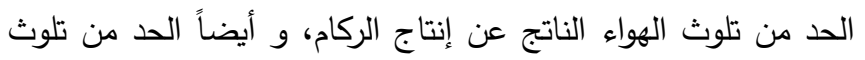
المركبات أثناء نقل المواد من مكان الصناعة الى نقطة الاستهلاك.

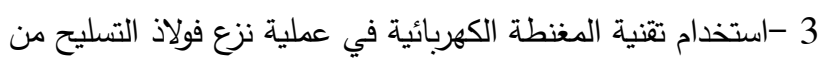

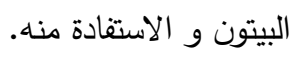
4 -تقليص مساحة المطامر المستخدمة للتخلص من النفايات، و إذا صار الطلب على المخلفات الليتونية أكبر فإنه يمكن فتح مناطق الردم القديمة وإعادة تدوير المخلفات البيتونية فيها ،و هذا من شأنه أن يعيد تأهيل

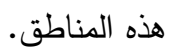
5 -الوفر الاقتصادي في المشروع من خلال تخفيض المال للازم لتأمين الحصويات، على سبيل المثال في إحدى الدراسات التي أجريت على

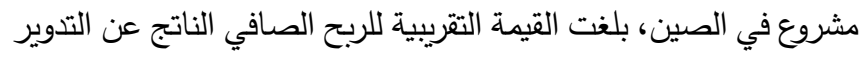
حوالي ال 2.5\% من ميزانية المشروع الكلية [3] . في الولايات المتحدة الامريكية سنوياً يتم تدوير 140 مليون طن 
4 -الحصويات المعاد تدويرها ( الأنقاض البيتونية):

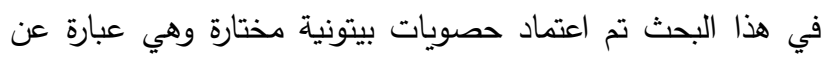
أنقاض بيتونية فقط تم الحصول عليها من الأبنية المهامة من عدة أحياء في محافظة حص ، حيث تم إحضارها إلى مخبر مديرية الخدمات الفنية

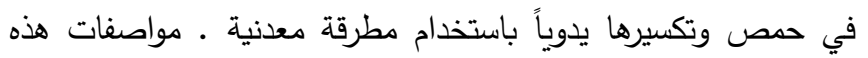

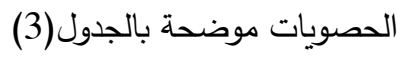
الجدول(3) الخصائص الفيزيائية و الميكانيكية للحصويات المعاد تدوير ها الماتيات

\begin{tabular}{|c|c|c|c|c|}
\hline الامتصاص \% & النعومة & النو الوزن & الحجمي & التدرج الحبي \\
\hline 7.4 & 6.5 & 2.6 & 1102 & $0.075-20$ \\
\hline
\end{tabular}

7. تصميم الخلطات البيتونية:

يبين الجدول (4) الكميات و الترميز المعتمد للخلطات البيتونية الخمس

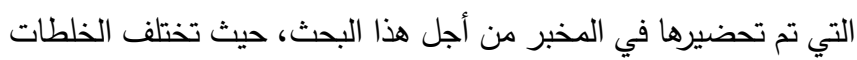

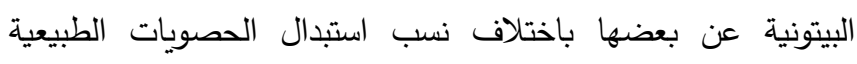

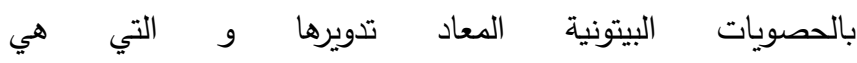

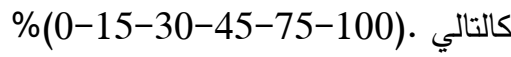
يثير الرمز SMM إلى الخلطة البيتونية المحضرة باستخدام حصويات طبيعية، أما الرمز M15 فيشير الى الخلطة البيتونية المصضرة باستخدام

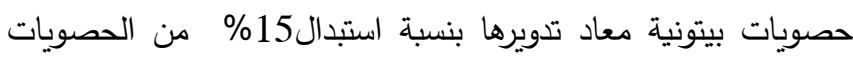
الطبيعية، و هكذا. تم استخدام عيار اسمنت في جميع الخلطات البيتونية. و تم اعتماد طريقة فوليير تومسون 5

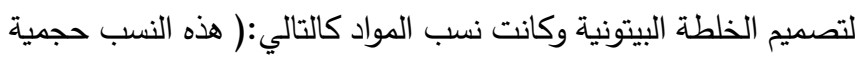

رمل قرواني 15\% رمل خشن 35\% بحص ناعم 20\% بحص خشن 30\% . خمو

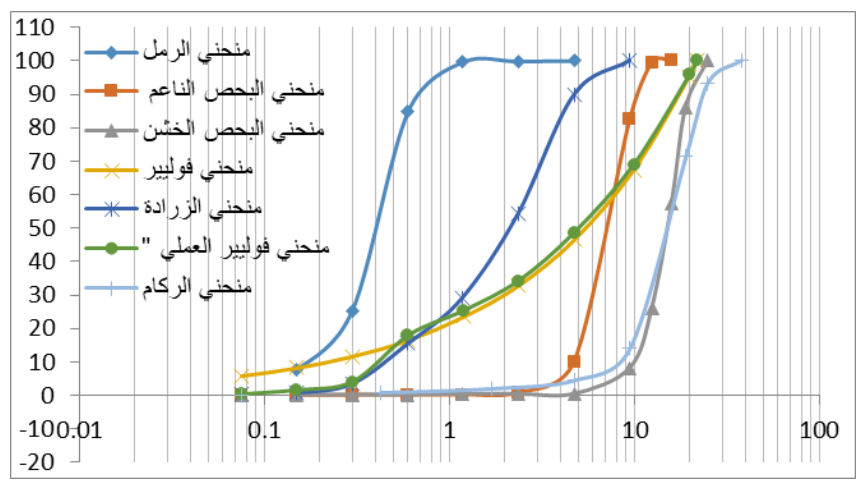

الثكل (4) منحنيات التحليل الحبي للمو اد الداخلة في الخلطة البيتونية

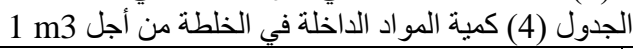

\begin{tabular}{|c|c|}
\hline النبب الوزنية للحصويات المعاد تدوير ها من وزن الحصويات & الخلطة \\
\hline 0 & SM \\
\hline 15 & M15 \\
\hline
\end{tabular}

-1-حماية الموارد الطبيعية.

-2-التتمية المستدامة على اساس بيئي.

-3-التخلص من الحجوم الضخمة للركام البيتوني الناجم عن الهدم.

\section{5. طرائق البحث و مواده : - 5}

اعتد البحث المنهجية التجرببية فيما يتعلق بدراسة الأنقاض البيتونية

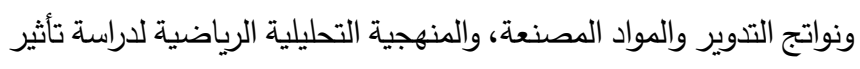
خصائص المواد المدورة في المنتجات المصنعة. وقد تم العمل وفق المراحل

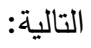

-1-الحصول على الأنقاض ومعالجتها مخبرياً) فرز - طحن - دراسة

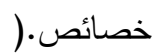

-2-توصيف الحصويات بعد تحضيرها كقياس التدرج الحبي والوزن

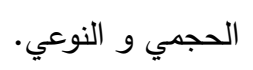

-3-صناعة النماذج المخبرية من البيتون و تحليل خواصها.

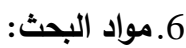

1 -الاسمنت : تم استخدام الصنف الأول من الاسمنت البورتلاندي

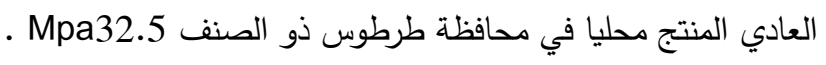

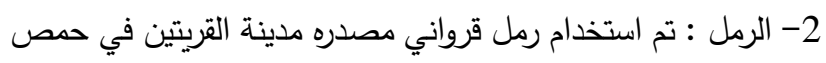

،وتم دراسة خصائصه الفيزيائية و الميكانيكية و النتائج موضحة فروني في في في

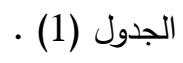

الجدول (1) الخواص الميكانيكةو الفيزيائية للارمل المستخذم.

\begin{tabular}{|c|c|c|c|c|}
\hline المكافئ & معامل & $\therefore$;ll & $\therefore$ idl & ح \\
\hline الرملي \% & النعومة & النوعي & الحجمي & الحبي \\
\hline 60 & 1.83 & 2.5 & 1474 & $0-2.36$ \\
\hline
\end{tabular}

3- الحصويات الطبيعية (رمل خشن و بحص ناعم و بحص خشن): تم استخدام الحصويات الطبيعية التي مصدرها كسارات مدينة حسياء في محافظة حمص ، حيث تم دراسة خصائصها الفيزيائية و الميكانيكية و

النتائج موضحة في الجدول(2) النمة

\begin{tabular}{|c|c|c|c|c|c|}
\hline \multicolumn{6}{|c|}{ الجدول (2) الخواص الميكانيكة و الفيزيائية للحصويات الطبيعية } \\
\hline مععامل & $\begin{array}{c}\text { الامتصناص } \\
\text { أنسبة }\end{array}$ & النوعي & الحجمي & التدرجي & \\
\hline 4.1 & 6 & 2.5 & 1550 & $0-4.75$ & خشن \\
\hline 6 & 3.5 & 2.67 & 1408 & $0-12.5$ & ناعم \\
\hline 7 & 3 & 2.69 & 1373 & $0-19$ & خشن \\
\hline
\end{tabular}




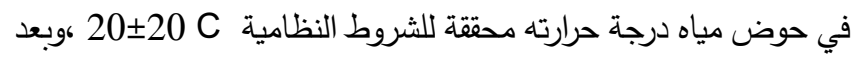
مرور 7 ايام تم اختبار 3 عينات ،و من ثم بعد 28 يوم تم اختبار باقي العينات لمعرفة مقاومتها على الضغط البسيط.

\section{9. - النتائج و مناقشتها :}

الخواص الفيزيائية :

- إن الخواص الفيزيائية للحصويات البيتونية المعاد تدويرها تختلف عن الخواص الفيزيائية للحصويات الطبيعية، وكما توضح الجداول السابقة فإن الوزن الحجمي للحصويات البيتونية المعاد تدويرها أقل من الوزن الحجمي للحصويات الطبيعية ، بينما ازدادت نسبة الامتصاص للحصويات البيتونية المدورة عن الحصويات الطبيعية ،و تعود أسباب اختلاف هذه الخصائص الى المونة الإسمنتية القديمة (الملاط الاسمنتي ) التي بقيت عالقة على سطح الركام المعاد تدويره ،حيث أن مسامية هذد المونة تسمح للمياه باختراق المسام وبالتالي زيادة كمية الماء الممتصة و تقليل الورن الحجمي لردي

$$
\text { - الوزن الحجمي للبيتون: }
$$

يعطي الجدول (5) قيم الأوزان الحجمية المحسوبة في الحالة الجافة على عمر 7 و 28 يوم ،حيث انخفضت قيم الوزن الحجمي بازدياد نسبة استبدال

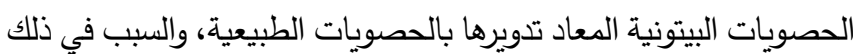
ايضا المونة الإسمنتية الملتصقة بسطحه. الجدول (5) قيم الاوزان الحجمية للبيتون

\begin{tabular}{|c|c|c|c|c|c|c|}
\hline 100 & 75 & 45 & 30 & 15 & 0 & الاستبدالب \\
\hline 2308 & 2351 & 2395 & 2408 & 2435 & 2443 & الجاف على الحمي \\
\hline
\end{tabular}

يوضـح الشكل(6) انخفاض الوزن الحجمي الجاف بازدياد نسبة استبدال الحصويات البيتونية بالطبيعية، حيث انخفض الوزن الحجمي الجاف للبيتون ذو الحصويات المعاد تدويرها بنسبة 100\% بمقدار يعادل5.5\% عن البيتون الطبيعي. المقاومة على الضغط البسيط : يوضـح الجدول (6) قيم المقاومات على الضغط للعينات الإسطوانية على عمر7 7 ايام و 28 يوم للبيتون ذي الحصويات المعاد تدويرها بالنسب \%(100-75-45-30-15) مقارنةً مع البيتون ذي الحصويات الطبيعية ،حيث تتخفض مقاومة البيتون بزيادة نسبة استبدال الحصويات كما هو مبين في الجدول حيث كل قيمة هي عبارة عن وسطي مقاومة الضغط لثلاث مقاومات أسطوانية محولة.

\begin{tabular}{|c|c|c|c|}
\hline \multicolumn{3}{|c|}{30} & M30 \\
\hline \multicolumn{3}{|c|}{45} & M45 \\
\hline \multicolumn{3}{|c|}{75} & M75 \\
\hline \multicolumn{3}{|c|}{100} & M100 \\
\hline \multicolumn{4}{|c|}{ 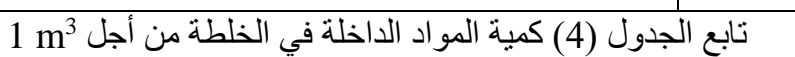 } \\
\hline وزن الماء 1 & وزن الاسمنت & 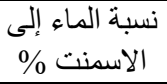 & الخلطة \\
\hline 189 & \multirow{6}{*}{350} & 0.54 & SM \\
\hline 196 & & 0.56 & M15 \\
\hline 203 & & 0.56 & M30 \\
\hline 203 & & 0.58 & M45 \\
\hline 210 & & 0.60 & M75 \\
\hline 217 & & 0.62 & M100 \\
\hline
\end{tabular}

تابع الجدول (4) كمية المو اد الداخلة في الخلطة من أجل 1 أل

\begin{tabular}{|c|c|c|c|c|c|}
\hline $\begin{array}{l}\text { وزنام } \\
\text { kg } \\
\text { kg }\end{array}$ & الخشن وزن البحن kg & 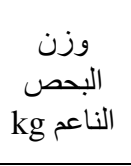 & 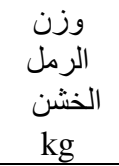 & وزن الرزمل & الخلطة \\
\hline 0.00 & 578.62 & 382.89 & \multirow{6}{*}{627.38} & \multirow{6}{*}{268.88} & SM \\
\hline 135.67 & 491.83 & 325.46 & & & M15 \\
\hline 266.99 & 405.03 & 268.02 & & & M30 \\
\hline 432.68 & 318.24 & 210.59 & & & M45 \\
\hline 721.13 & 144.66 & 95.72 & & & M75 \\
\hline 961.51 & 0.00 & 0.00 & & & M100 \\
\hline
\end{tabular}

ملاحظة : تم زيادة نسبة الماء إلى الاسمنت بزيادة نسبة الاستبدال و ذلك من أجل الحفاظ على قابلية تثغيل ثابتة و مقبولة ( قيمة الهبوط في مخروط أبرامز 5cm). 8. تحضير و صب العينات:

تم خلط الحصويات البيتونية المعاد تدويرها مع الحصويات البيتونية الطبيعية ثم إضافة الرمل ومن ثم الإسمنت إلى الخليط، حيث تم الخلط في حوض جبالة بيتونية في المخبر ، بعد ذلك تمت إضافة الماء إلى الخليط، واستمر الخلطحتى تجانس المزيج، ومن ثم أجريت اختبارات قياس قابلية التشغيل ثلاث مرات لكل خلطة وذلك باستخدام مخروط أبرامز • بعد هذا تم خلط البيتون مرة أخرى ومن ثم صب 6 عينات مكعبية بأبعاد 200*200 mm ) (200 ) لكل نسبة استبدال وذلك في قوالب معدنية ورصها بواسطة قضيب معدني، ثم تم فك القوالب بعد يوم كامل ووضعها 


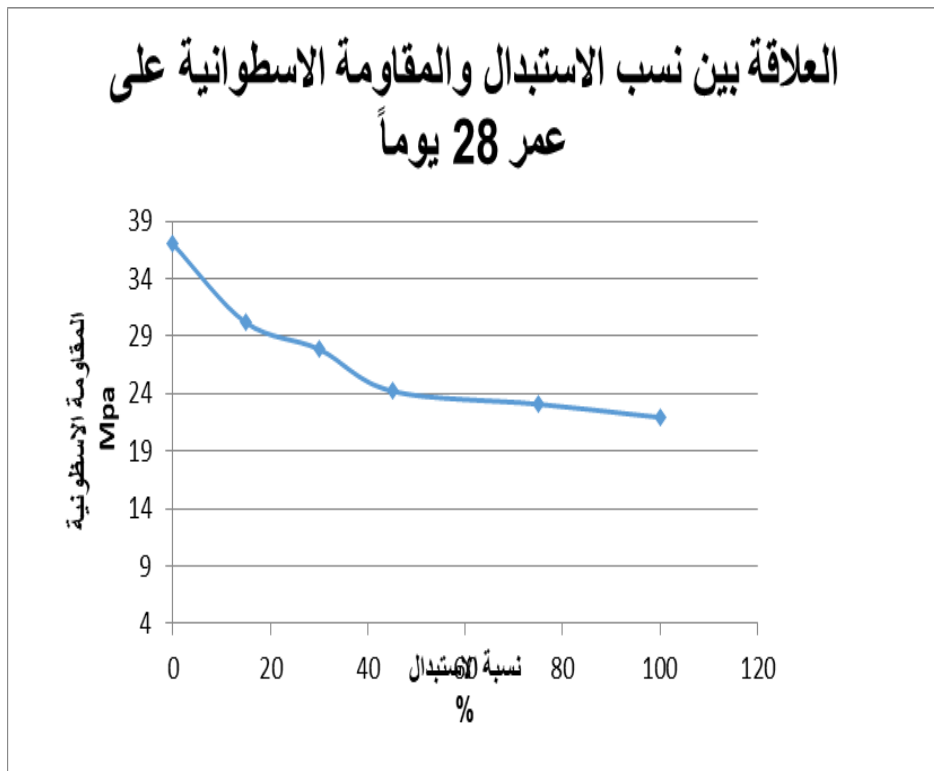

الثكل (7) العلاقة بين نسبة الاستبدال و المقاومة على الضغط البسيط

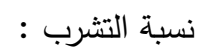

تابع الجدول (6) قيم المقاومات على الضغط للعينات الأسطوانية على عمر 7 و 28 يوم

\begin{tabular}{|c|c|c|c|c|}
\hline 30 & $\rho$ & 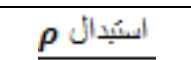 & & \\
\hline$\frac{\rho+0}{\rho 7}$ & 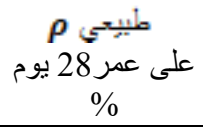 & 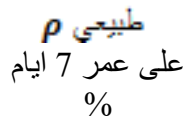 & \% التشرب & الاستبدال \\
\hline 1.50 & 1.00 & 1.00 & 5.50 & 0 \\
\hline 1.48 & 0.92 & 0.93 & 5.90 & 15 \\
\hline 1.41 & 0.81 & 0.85 & 6.23 & 30 \\
\hline 1.31 & 0.71 & 0.81 & 6.50 & 45 \\
\hline 1.23 & 0.63 & 0.77 & 6.83 & 75 \\
\hline 1.28 & 0.58 & 0.68 & 7.12 & 100 \\
\hline
\end{tabular}

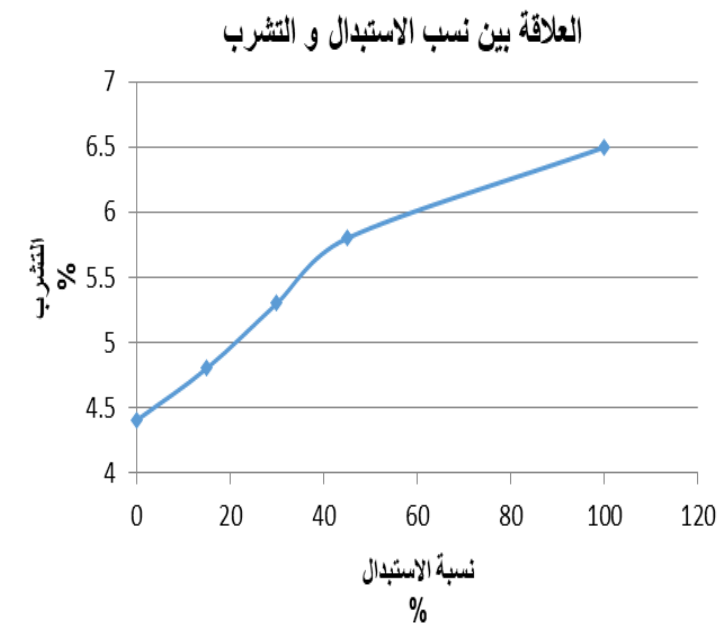

قهنا بتمثيل العلاقة بين مقاومة العينات على الضغط البسيط ونسب الاستبدال وذلك على الثكل (7) يوضح الثكل(8) العلاقة بين التشرب ونسب استبدال الحصويات الطبيعية

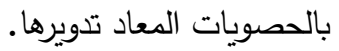

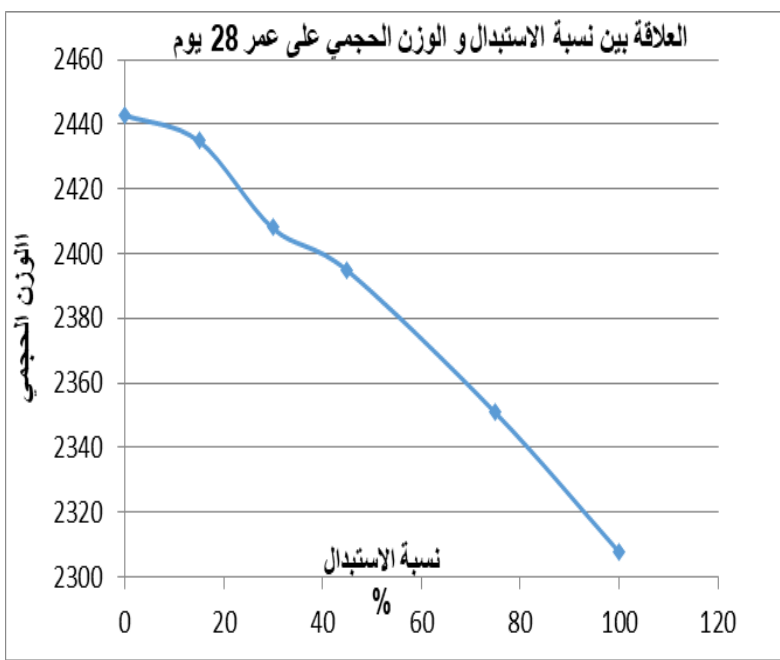

الثكل (6) العلاقة بين نسبة الاستبدال و الوزن الحجمي الجدول (6) قيم المقاومات على الضغط للعينات الأسطوانية على عمر 7

\begin{tabular}{|c|c|c|}
\hline 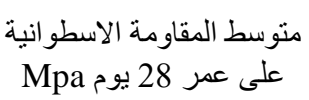 & متلى عمر المقاومة الاسطو انية 7 ايام Mpa & الاستبدال \% \\
\hline 33.12 & 22.08 & 0 \\
\hline 30.42 & 20.53 & 15 \\
\hline 26.70 & 18.86 & 30 \\
\hline 23.40 & 17.90 & 45 \\
\hline 20.90 & 17.02 & 75 \\
\hline 19.20 & 15.00 & 100 \\
\hline
\end{tabular}

إذ تسجل العينات ازدياداً في تشربها من النسبة 5.50 للعينات دون استبدال ( حصويات طبيعية ) إلى النسبة 7.12 للعينات المنفذة كليا مَّن الحصويات المعاد تدويرها، يعود ذلك لنفس السبب المشروح سابقا وهو امتصاص الحصويات المدورة للماء ـ سيحتاج المهندس عند استخدام هذه

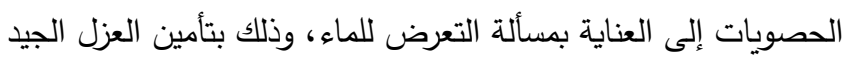
للبيتون، أو إضافة بعض الإضافات البيتونية التي تسىى في مجال مواد

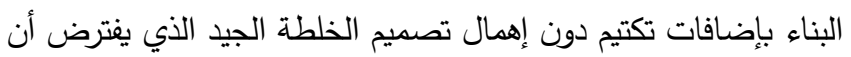

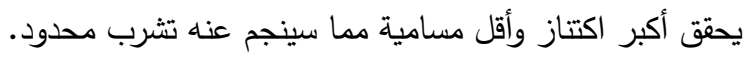


- من خلال الرجوع إلى الدراسات العالمية استطعنا الوصول إلى دراسة

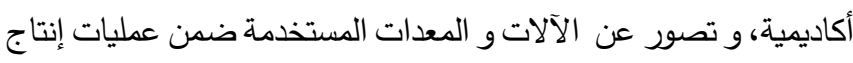
الحصويات الطبيعة و الحصويات المعاد تدويرها، كذلك تم الحصول على الحى مواصفات تلك الآلات من حيث طاقتها الإنتاجية و العدد اللازم من كل آلة. نبين في الجدولين (7) و (8) مواصفات الآلات المستخدمة في إنتاج الحصويات الطبيعية و الحصويات المعاد تدويرها . الجدول (7) مواصفات الآلات المستخدمة و اللازمة لإنتاج الحصويات

\begin{tabular}{|c|c|c|c|c|c|}
\hline \multicolumn{6}{|c|}{ الطبيعية } \\
\hline العناصر & $\begin{array}{c}\text { الآلة اللة } \\
\text { KW }\end{array}$ & اسم الآلة & & |المرحلة & المرحلة \\
\hline 2 & 238.6 & تركس جنزير & $\mathrm{A} 1$ & \multirow{5}{*}{ المواد الطتخراج } & \multirow{5}{*}{1} \\
\hline 3 & 287 & هيدروليكية & $\mathrm{A} 2$ & & \\
\hline 2 & 162 & تركس دواليب & $\mathrm{A} 3$ & & \\
\hline 6 & 179 & شاحنة الكفاءة & A4 & & \\
\hline 1 & 72 & الاستخدامات حفارة متعددة & A5 & & \\
\hline 1 & 165 & كسارة اساسية & A6 & \multirow{8}{*}{ فرن فرير و } & \multirow{8}{*}{2} \\
\hline 1 & 195 & كسارة صدمية & A7 & & \\
\hline 2 & 16 & لعملية الغربلة & A8 & & \\
\hline 3 & 19 & غربال & A9 & & \\
\hline 1 & 18 & الر لغسل & A10 & & \\
\hline 3 & 20 & الة لغسل & A11 & & \\
\hline 1 & & لوحة تحكم & $\mathrm{A} 12$ & & \\
\hline 16 & 11 & سيور ناقلة & A13 & & \\
\hline
\end{tabular}

في كلا الجدولين نلاحظ أن المرحلة الأولى تعتمد على معدات الديزل، بينما

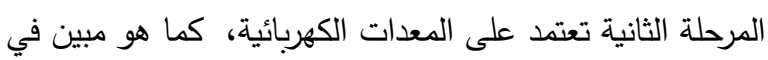

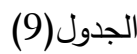
- الطاقة التي تستهلكها المعدات ( ديزل و كهرباء ) تسمى الطاقة النهائية

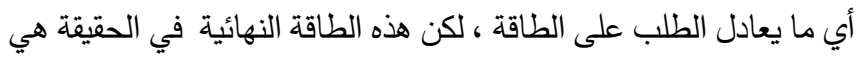
نتاج مصدر أولي يعرف باسم الطاقة الأولية .
الشكل (8) العلاقة بين نسبة الاستبدال و التشرب

10. الاستتتاجات لهذا الجزء من البحث :

يمكن تسجيل النقاط التالية كاستتتاجات هامة لهذا البحث: -1-تعطي الحصويات المعاد تدويرها قيم مقبولة للمقاومات في البيتون عند استخدامها بنسب مختلفة .وهو مايفسح المجال واسعاً للتفكير باستخدامها في بيتون المنشآت المدنية.

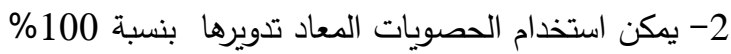

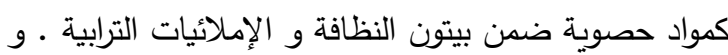
يمكن استخدام نسبة 75\% ضمن أعمال البيتون الإنشائي . -3- تظهر أغلب المنحنيات (التي تعبر عن تغير مواصفات البيتون بدلالة نسب الاستبدال) انسيابية يبدو معها تأثير استبدال الحصويات الطبيعية بالحصويات المعاد تدويرها غير حاد ولا يغير مواصفات البيتون المختلفة بشكل جذري. -4- من أجل الحفاظ على قابلة تشغيل مقبولة ، تم زيادة نسبة الماء الى هويل الإسمنت مع زيادة نسبة الحصويات المدورة، ( من أجل الخلطة كSM مانت

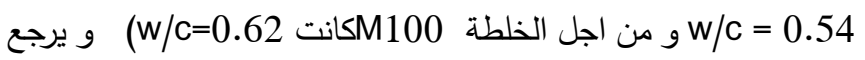
ذلك إلى المونة الإسمنتية العالقة على سطح الحصويات المعاد تدويرها والتي تزيد من قابلية امتصاصسه.

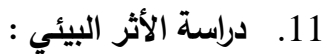

تم في هذا البحث افتراض وجود ثلاث اتجاهات و إجراء مقارنة بيئية بينهما : الأول : إنتاج الحصويات الطبيعة (NAtural Aggregate) (NA)

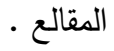
الثاني: إنتاج الحصويات المعاد تدويرها من البيتون المهدم Recycled) . concrete Aggregate)(RCA) الثالث : إنتاج خلطة بيتونية بنسبة 75\% من الحصويات المعاد تدويرها

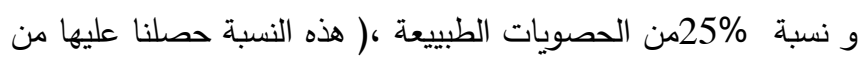
التجارب السابقة التي قمنا بها على الخلطات البيتونية الداخل في تركيبها حصويات بيتونية معاد تدويرها ).

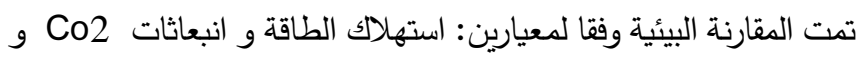
ذلك من خلال دراسة دورة حياة هذه المواد ، و تم اختيار هذين المعيارين كونهما يعدان من الجوانب الرئيسية تجاه القضايا البيئية في مجال البناء ـ - بشكل عام إن انتاج الحصويات الطبيعية يتكون من مرحلتين :

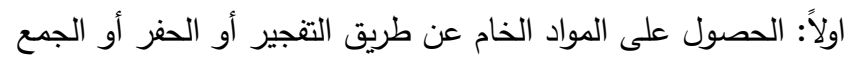
بينهما. ثانياً: نقل الصخور إلى مصنع التكسير حيث يتم هناك تكسير الحصويات

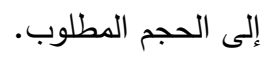
- إن تكنولوجيا إنتاج الحصويات المعاد تدويرها يشبه إنتاج الحصويات الطبيعية، و لكن اجراءات التكسير و إعادة التدوير و الآلات المستخدمة تختلف قليلاً . 
، فيما يلي نستعرض الصيخ الرياضية المستخدمة لتقدير استهلاك الطاقة و انبعاثات Co2 في كل من الاتجاهين المعتمدين .

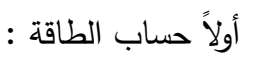
الاستهلاك النهائي من الطاقة ( الطلب على الطاقة ) من كل مرحلة يتم

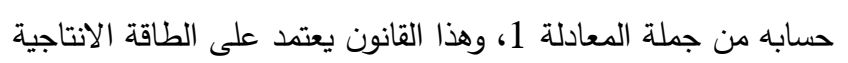
للآلات و العدد اللازم منها. الجدول (9) عوامل التحويل المستخدمة في الدراسة و هذه الارقام وفقاً

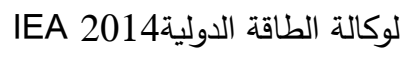

\begin{tabular}{|c|c|c|c|c|}
\hline تمعامل & تحول & الطاقة & المرحلة & \\
\hline 3.032 & 0.086 & ديزل & استخراج & \multirow[t]{2}{*}{ الاتجاه الأول } \\
\hline 1.2 & 0.29 & كهرباء & غربلة و & \\
\hline 3.032 & 0.086 & ديزل & تحضير & \multirow[t]{2}{*}{ الاتجاه الثاني } \\
\hline 1.2 & 0.29 & كهرباء & غربلة و & \\
\hline 3.032 & 0.086 & ديزل & و استخراج & \multirow[t]{2}{*}{ الاتجاه الثالث } \\
\hline 1.2 & 0.29 & كهرباء & غربلة و & \\
\hline
\end{tabular}

الجدول(10 )الرموز المستخدمة في الدراسة

\begin{tabular}{|c|c|}
\hline 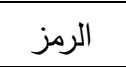 & التوصيف \\
\hline$A i$ & الآلة i \\
\hline $\mathrm{Pi}$ & استطاعة الآلة i \\
\hline$R$ & 200ton/h الطاقة الإنتاجية للمصنع تم فرضها \\
\hline$N i$ & e عد العناصر المطلوبة من الجهاز \\
\hline$n$ & عدد الأجهزة في الخطوة 1 \\
\hline$m$ & عدد الأجهزة في الخطوة 2 \\
\hline FED & Kwh/ton استهلاك الطاقة النهائي = الطلب النهائي على الطاقة \\
\hline PED & الطلب الرئيسي على الطاقة(kgoe/ton) \\
\hline COE & انبعاثات ثاني أكسيد الكربون الدحدة (kg CO2eq/ton) \\
\hline FPEF & معامل تحويل الطاقة \\
\hline FCOE & معامل تحويل الانبعاثات \\
\hline
\end{tabular}

- في هذه الدراسة: تم حساب الطاقة الأولية انطلاقاً من الطاقة النهائية ،

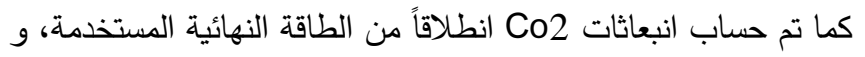
ذللك بناء على العوامل الواردة في الجدول (9). الجدول (8) مواصفات الآلات المستخدمة و اللازمة لإنتاج الحصويات لإلات

\begin{tabular}{|c|c|c|c|c|c|}
\hline \multicolumn{6}{|c|}{ المعاد تدويرها } \\
\hline العناصر & $\begin{array}{c}\text { استطاعة الآلة } \\
\text { KW }\end{array}$ & اسم الآلة & & المرحلة & المرحلة \\
\hline 1 & 107 & هيدروليكية & $1 \mathrm{~A}$ & تحضير & 1 \\
\hline 1 & 162 & تركس & $\mathrm{A} 2$ & & \\
\hline 6 & 179 & شاحنة & $\mathrm{A} 3$ & & \\
\hline 1 & 90 & كسارة & $\mathrm{A} 4$ & & \\
\hline 1 & 250 & صدارة & A5 & تكسير و & \\
\hline 1 & 15 & رجاجات & A6 & فرز & \\
\hline 1 & 30 & غربال & A7 & & 2 \\
\hline 1 & 3 & لفناطيسي & A8 & & \\
\hline 2 & 5.5 & 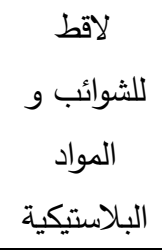 & A9 & & \\
\hline 1 & 83 & فلتر للغبار & $\mathrm{A} 10$ & & \\
\hline 1 & & لوحة تحكم & $\mathrm{A} 11$ & & \\
\hline 11 & 7.5 & سيورناقلة & A12 & & \\
\hline
\end{tabular}

- المصطلحات والتعاريف المستخدمة في هذه الدراسة مبينة في الجدول( 10 ) ، حيث الكيلو غرام مكافئ من النفط واحدة تستخدم لحساب الطاقة،

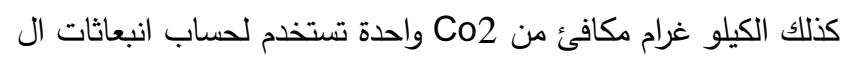
.Co2 
12. دراسة الجدوى الاقتصادية :

تم في هذا القسم أيضاً المقارنة بين حلين :

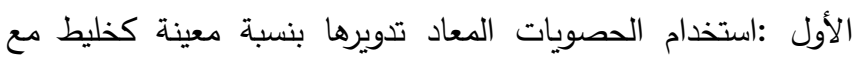
الحصويات الطبيعية ضمن الأعمال المدنية ( و هذا ما قمنا بإجراء

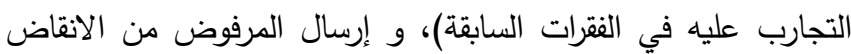

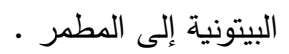
الثاني: إرسال كافة الانقاض المهدمة الى المطمر ، و استخدام الحصويات الطبيعية فقط ضمن الأعمال المدنية . ملاحظة : جميع الارقام الواردة في هذه الدراسة وفقاً لمديرية الخذمات الفنية

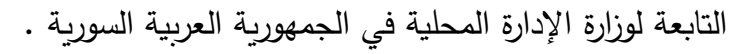

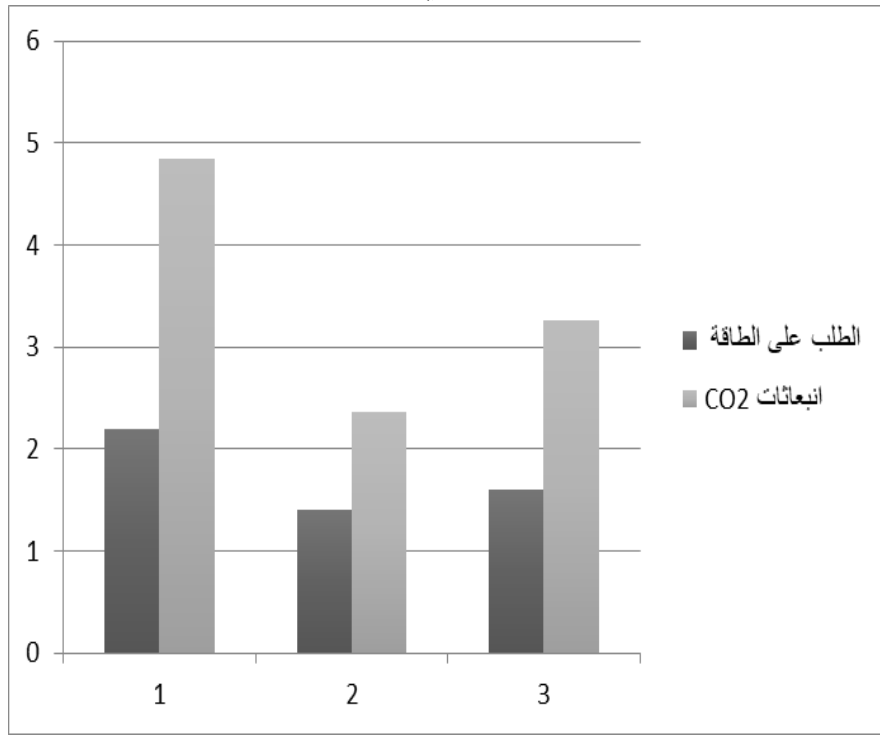

الثكل (9) مقارنة بيئية بين الاتجاهات الددروسة

اولا الدراسة الاقتصادية للحل الأول : إن عملية إعادة التدوير تقسم الى ثلاث مراحل :

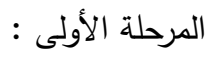

أعمال الهام و تكسير الكتل الكبيرة و نقل و تجميع نواتج الهدم إلى موقع

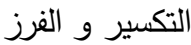

مجموع كلف الهـ مع الترحيل الى مواقع التكسير \$ \$ 3.5

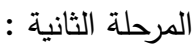
تكسير الكتل و فرز المواد الناتجة و سحب الحديد وتجميع المواد الصالحة للاستخدام و نقل المواد المرفوضة إلى المطامر العامة.

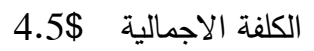

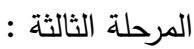
نقل المواد الحصوية بعد تصنيفها و اختبارها الى مواقع الاستخدام. الكلفة الاجمالية \$ بناء على ماسبق يمكن حساب تكلفة المتر المكعب الواحد من نواتج التدوير ، و ذلك لاستخدامه كمواد حصوية ضمن أعمال البيتون الإنشائي اعتماداً على النسبة التي حصلنا عليها من التجارب السابقة.

$$
\text { FEDstep } 1=\sum_{i=1}^{n} \frac{P i * N i}{R}
$$

$$
\text { FEDstep } 2=\sum_{i=n+1}^{m} \frac{P i * N i}{R}
$$

وفقا للمعادلة 2 إجمالي استهلاك الطاقة الأولية في كل اتجاه يساوي

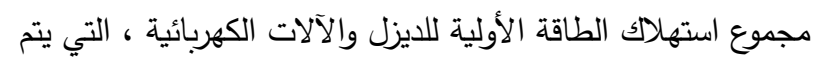
الحصول عليها عن طريق ضرب طاقتهم النهائية بعامل تحويل الطاقة من الجدول (9) - معتمل PEDtotal $=$ PEDstep1+ PEDstep2 $=($ FED*FPEF $)$ step1 $+($ FED*FPEF)step2

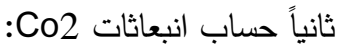
وفقا للمعادلة (3 ) إجمالي انبعاثات ثاني اكسيد الكربون في كل اتجاه يتم حسابه عن طريق جمع انبعاثات Co2 لكل مرحلة ، و التي نحصل عليها

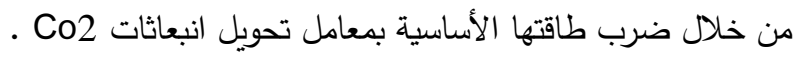
COEtotal $=$ COEstep $1+$ COEstep $2=$ (PED*FCOE)step1+(PED*FCOE)step2(3)

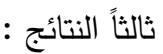
بتطبيق المعادلات السابقة وفقا للمعطيات الواردة في الجدولين 1 و 2 نحصل على النتائج المبينة في الجدول (11)

\begin{tabular}{|c|c|c|c|}
\hline $\begin{array}{c}\text { انبعاثات } \\
\text { CO2 } \\
\text { Kg co2eq } \\
\text { /ton }\end{array}$ & $\begin{array}{l}\text { الطاقة الأولية من } \\
\text { Kgoe/ton }\end{array}$ & المعالجة & الاتجاه \\
\hline 3.66 & 1.21 & استخراج & \multirow{3}{*}{ الالجاه } \\
\hline 1.19 & 0.99 & غربلة و & \\
\hline 4.85 & 2.2 & المجموع & \\
\hline 1.75 & 0.58 & تحضير & \multirow{3}{*}{ الاتجاه } \\
\hline 0.98 & 0.82 & غربلة & \\
\hline 2.73 & 1.4 & المجموع & \\
\hline 2.23 & 0.74 & تحضير & \multirow{3}{*}{ الاتجاه } \\
\hline 1.03 & 0.86 & غربلة و & \\
\hline 3.26 & 1.6 & المجموع & \\
\hline
\end{tabular}
الجدول (11) نتائج الدراسة البيئية 


\begin{tabular}{|l|l|l|l|l|}
\hline 33.33 & 12 & $11-3=8$ & m3 & $\begin{array}{r}\text { مواد حصوية لتصنيع } \\
\text { بيتون نظافة و إملاء }\end{array}$ \\
\hline
\end{tabular}

الجدول (12) نتائج الدراسة الاقتصادية

13. تحليل النتائج : n

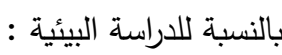

1- كثفت النتائج أن الاتجاه الأول يتسبب بنسبة أعلى من استهلالك الطاقة

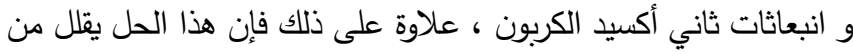

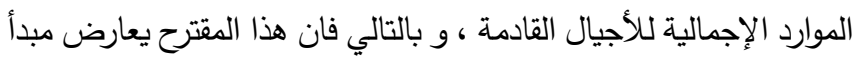

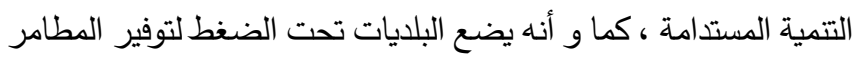

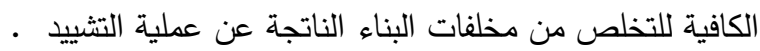
2- يعد الاتجاه الثاني الأفضل بيئياً كونه يخفف من استهلاك الطاقة و انبعاثات Co2 بنسبة 37\% و 44\% على التوالي مقارنة مع السيناريو الأول ، كما و أنه يخفف من المساحات اللازمة لطمر المخلفات البيتونية

وبالتالي حماية المساحات الخضراء و و حماية البيئة . 3- وفقاً للنتائج التي حصلنا عليها في الفصل الرابع عبرنا عنها بالاتجاه الثالث فإن استخدام هذه النسبة سوف يخفف من استهلاك الطاقة و

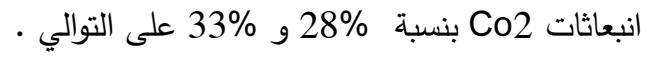
بالنسبة للدراسة الاقتصادية : 4- بلغت نسبة التوفير الاقتصادي من استخدام الحصويات المعاد تدويرها في أعمال البيتون الإنثائي ما يقارب ال 44\% ، و النسبة الناتجة من النافي استخدام تلك الحصويات في تصنيع بيتون النظافة والإملائيات الترابية ما

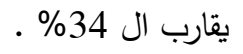
5- في بعض الأحيان تكون تكلفة إنتاج الحصويات المعاد تدويرها مرتفعة نوعاً ما مقارنة بالحصويات الطبيعية، و لكن يمكن بسهولة تعويض التكلفة الزائدة عن طريق جوانب أخرى من الفوائد، حيث أنه لا يمكن قياس التحليل بهذه البساطة و ربطه فقط بتكلفة الإنتاج و المؤشر الاقتصادي فقط ، و لكن يجب دمج التحليل الاقتصادي مع الفوائد الاجتماعية و البيئية 13- التوصيات : - إن أي نفايات ناتجة عن النشاط البنائي يجب أن نتعامل معها بالثكل الصحيح حيث يجب ألا يتم القاءها بثكل تعسفي ،و يجب على الحكومة

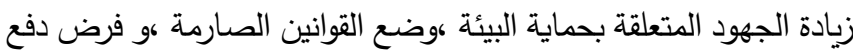
الغرامات و استخدام السياسات الضريبية لتشجيع إعادة تدوير مخلفات

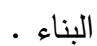

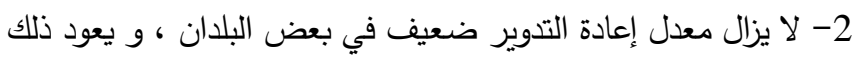

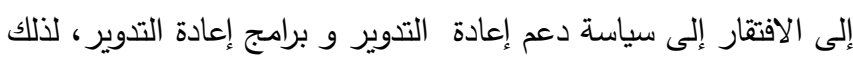

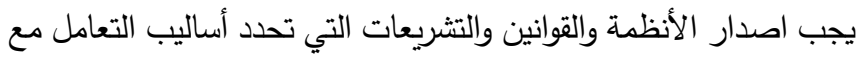

هذه الكلفة تتضمن 75\% (كلفة الهام + كلفة التكسير و الفرز) +25\% (ثمن المواد الطبيعية ) حيث تبلغ ثمن المتر المكعب من المواد الحصوية الطبيعة \$3و ثمن نقلها

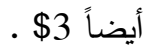
$(3.5+4.5+3) * 0.75+(6 * 0.25)=9.75 \$$ ووفقاً لنتائجنا السابقة فإنه يمكن استخدام الحصويات المعاد تدويرها بنسبة 100\% في أعمال البيتون غير الإنثائي ( بيتون نظافة - إملاء ) و و

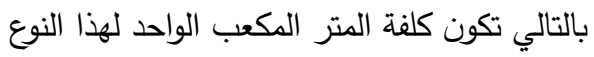
$4.5+3.5+3=11 \$$ الدراسة الاقتصادية للحل الثاني : يتضمن الحل التاني إرسال كل الأنقاض المدمرة الى المطمر ، و استخدام

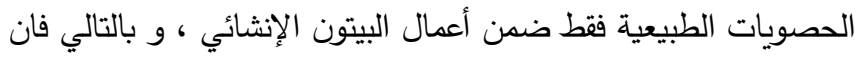
هذه الحل يتضمن كلفة هدم و ترحيل الأنقاض و كلفة الطمر و التسوية و كلفة إنتاج الحصويات الطبيعية . و بالتالي كلفة المتر المكعب الواحد تتضمن كلفة الهام (3.5) +كلفة الترحيل لكامل الهمميات للمقالب العامة (4.5) + ثمن الحصويات الطبييعة

بأرض الكسارة (6) $3.5+4.5+6=14 \$$ نبين في الجدول (12) المقارنة بين النتائج التي حصلنا عليها أي بين كلفة الهدم و التكسير و الفرز (و استخدام الناتج مطروحاً منه قيمة حديد التسليح

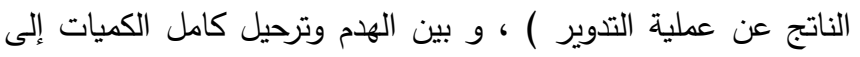
الدكبات و استخدام كامل كميات المواد الحصوية من الخامات الطبيعية حسب مجال الاستخدام ، و ذلك بفرض أن كمية حديد التسليح المستخرج من الأنقاض بمعدل 20Kg/m3 و سعر ال كغ الواحد من حديد النفايات 0.15\$و بالتالي تكون قيمة الحديد:

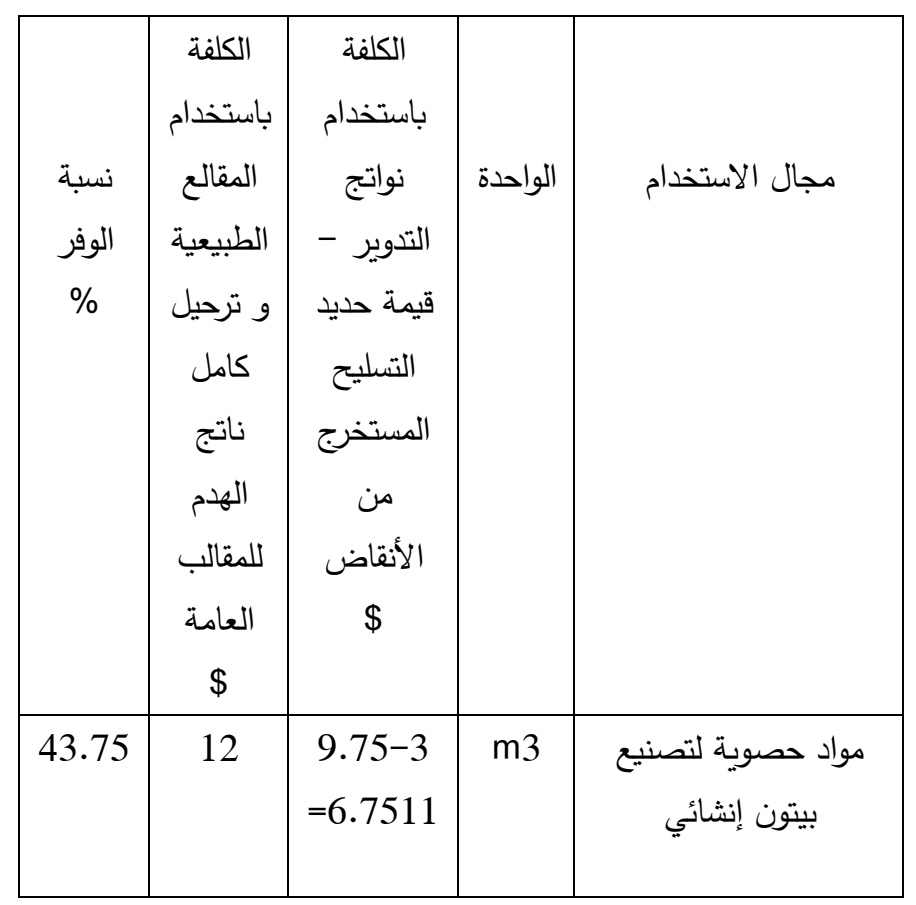


مخلفات البناء والهدم على أن تتضمن تشجيع أساليب الخفض من المصدر وإعادة الاستخدام وإعادة التدوير مع الحد من التخلص منها في المطامر .

3-بعد النتائج الهامة التي خلص إليها البحث من حيث صلاحية أنقاض البيتون المهام كلياً أو جزئياً لصناعة البيتون، لابد من إيلاء مكبات

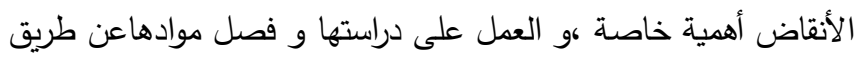
محطات خاصة بإعادة التدوير تمهيداً لاستخدامها في المجابل البيتونية للالك يجب تشجيع تأسيس مصانع ومعامل مركزية لإعادة تدوير مخلفات البناء والهدم في المدن الكبرى.

4- إقامة مؤتمرات طبية متخصصة لدراسة مدى خطورة أنقاض البناء

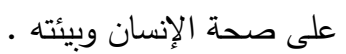

5- التعاون مع الإعلام السمعي والمرئي لتوعية المواطنين بخطورة الأنقاض بيئياً

6- إجراء المزيد من الأبحاث لتحسين سلوك البيتون الذي يحتوي على حصويات معاد تدويرها،و ذلك بمحاولة الحصول على حصويات بيتونية بجودة عالية أو من خلال استخدام إضافات كالألياف، أو المواد البوزولانية وغيرها.

7- من أجل الحفاظ على نسبة ماء إلى اسمنت مقبولة لا تؤثر على مقاومة البيتون يمكن استخدام الملدنات فائقة الأداء.

\section{قائمة المصادر و المراجع :}

[1] PAUL, S-2011- "Mechanical Behaviour And Durability Performance Of Concrete Containing Recycled Concrete Aggregate", South Africa.

[2] Rao .A Jba , K .N .and Misra S - 2007- "Use of aggregate from recycled construction and demolition waste in concrete", Conservation and Recycling, Journal of Resources.

[3] Akmal S. Abdelfatah and SamiW. Tabsh -2011- "Review of Research on and Implementation of Recycled Concrete Aggregate in the GCC" .

[4] Tomas U, Ganiron Jr -2010-" Recycling Concrete Debris from Construction and Demolition Waste College of Architecture", Qassim University.

[5] World Business Council for Sustainable Development- 2009 "The Cement Sustainability Initiative- Recycling Concrete" . 